PROCEEDING S OF THE

AMERICAN MATHEMATICAI. SOCIFTY

Volume 87. Number I. January 1983

\title{
ON MAXIMAL IDEALS DEPENDING ON SOME THIN SETS IN $M(G)$
}

\author{
ENJI SATO
}

\begin{abstract}
Let $M(G)$ be the convolution measure algebra on the LCA group $G$ with dual $\Gamma$. and $J$ the maximal ideal space of $M(G)$. For $E \subset G$ a compact set. let (ip $(E)$ be the subgroup of $(j$ generated algebraically by $E . R(E)$ the measures which are carried by a countable union of translates of $G p(E)$. and $P_{l}$ : the natural projection from $M(G)$ onto $R(E)$. Also let $h_{l}$ be the multiplicative linear functional $\mu \mapsto\left(P_{t} \mu\right)(1)$ on $M(G)$. Then we prove that if $G$ is an $l$-group. and $E$ an $H_{1}$-set, we get $h, \in \bar{\Gamma}$ (i.e. the closure of $\Gamma$ in $\Delta$ ).
\end{abstract}

1. Introduction. Let $G$ be a nondiscrete LCA group with dual $\Gamma$, and $M(G)$ the convolution measure algebra of $G\left(\mathrm{cf}\right.$. [8, 14]). We denote by $\Delta=\Delta_{M_{(G)}}$ the maximal ideal space of $M(G)$. Given a Borel set $E$ in $G$, let $I(E)$ be the set of those measures $\mu$ in $M(G)$ which satisfy $|\mu|(G p(E)+x)=0$ for all $x \in G$, where $G p(E)$ is the algebraic group generated by $E$, and $R(E)=I(E)^{\perp}$ be the set of those measures in $M(G)$ which are singular with respect to all members of $I(E)$. Thus $I(E)$ and $R(E)$ are an $L$-ideal and an $L$-subalgebra of $M(G)$, respectively, and $M(G)$ can be decomposed into the direct sum of $I(E)$ and $R(E)$. Moreover, each measure in $R(E)$ is carried by countable union of translates of $G p(E)$. Let $P_{E}$ denote the natural projection from $M(G)$ onto $R(E)$. Then $P_{E}$ is multiplicative and the linear functional $\mu \mapsto\left(P_{E} \mu\right) \hat{(1)}=\left(P_{E} \mu\right)(G)$ is a complex homomorphism of $M(G)$, which we will denote by $h_{E}$.

C. F. Dunkl and D. E. Ramirez [3] proved $h_{E} \in \bar{\Gamma}$ (i.e. the closure of $\Gamma$ in $\Delta$ ) for some Borel set $E$. In this paper we shall study $h_{E}$ for some thin sets $E$.

In order to prove our results, we need some notations. For $\mu \in M(G)$, we denote $\hat{\mu}$ the Gelfand transform of $\mu$ which equals to Fourier-Stieltjes transform on $\Gamma$, and $\|\hat{\mu}\|_{\infty}=\sup \{|\hat{\mu}(\gamma)|: \gamma \in \Gamma\}$. Given a set $K$ in $G$ and a natural number $n$, we define $n K=K+\cdots+K(n$ times $)$. Also we define $n K=\{0\}$ if $n=0$, and $n K=(-n)(-K)$ if $n$ is a negative integer. Given a subgroup $H$ of $G$, we shall define that $K$ is $H$-independent if (a) $K \cap H=\varnothing$, and if (b) whenever $x_{1}, \ldots, x_{n}$ are finitely many distinct elements of $K,\left(p_{1}, \ldots, p_{n}\right) \in \mathbf{Z}^{n}$, and $p_{1} x_{1}+\cdots+p_{n} x_{n} \in H$, then $p_{j} x_{j} \in H$ for all $j=1,2, \ldots, n$. Notice that when $H=\{0\}$, the above definition agrees with the usual definition (cf. [8]). We shall say that a LCA group $G$ is an $I$-group if every nonempty open set in $G$ contains an element of inifinte order. The author wishes to thank Professor S. Saeki for his many helpful criticisms and suggestions.

Received by the editors August 19, 1981.

1980 Mathematics Subject Classification. Primary 43A10; Secondary 43A46.

Kel' words and phrases. Measure algebra on LCA group, maximal ideal, Helson set.

(C) 1983 American Mathematical Society 0002-9939/82/0000-0622/\$02.50 
2. Maximal ideals and thin sets. Let $G$ be a LCA group and $K$ a compact subset of $G$. $K$ is called an $H_{\alpha}$-set $\operatorname{if} \inf \left\{\|\hat{\mu}\|_{\infty} \mid\|\mu\|=1, \mu \in M(K)\right\}=\alpha(0<\alpha \leqslant 1)$. We shall simply call $K$ a Helson set, if $K$ is an $H_{\alpha}$-set for some $\alpha$.

THEOREM 1. Let $G$ be a nondiscrete LCA I-group. Then if $K$ is an $H_{1}$-set, we have $h_{k} \in \bar{\Gamma}$. Also there exists $K$ and $H_{\alpha}$-set $(0<\alpha<1)$ such that $h_{K} \notin \bar{\Gamma}$.

THEOREM 2. Let $G$ be a nondiscrete LCA group. Then there exists $K$ a non-Helson set in $G$ such that

$$
\underset{\substack{\gamma \rightarrow \infty \\ \gamma \in \Gamma}}{\lim }\|\gamma-1\|_{K}=0 \text { and } h_{K} \notin \bar{\Gamma} .
$$

COROLlaRY 1 (cf. [1]). Let $G$ be a nondiscrete LCA I-group. Then there exists $\left\{h_{K}\right\}$ uncountable maximal ideals such that $h_{K} \in \Delta \backslash \bar{\Gamma}$, and there exist $\left\{h_{K}\right\}$ uncountable maximal ideals such that $h_{K} \in \bar{\Gamma} \backslash \Gamma$.

COROLlaRY 2. Under the notation of Theorem $2, h_{K}$ is not a strong boundary point for the uniform closure of $M(G)$ in $C(\Delta)$.

The next result is obtained by C. F. Dunkl and D. E. Ramirez [2].

Proposition. Let $f$ be an element in $\Delta$. Then $f$ is contained in $\bar{\Gamma}$ if and only if $|\hat{\mu}(f)| \leqslant\|\hat{\mu}\|_{\infty}$ for all $\mu \in M(G)$.

In order to prove our results, we need some lemmas.

LEMMA 1 . Let $K$ be an $H_{1}$-set with $K \ni 0$ in $G, \varepsilon$ a positive number, and $E$ a compact subset in $G$ with $E \cap G p(K)=\varnothing$. Then there exists a probability measure $\mu_{\varepsilon, E} \in$ $M(G)$ such that $\left\|\hat{\mu}_{\varepsilon, E}-1\right\|_{K}<\varepsilon$ and $\left\|\hat{\mu}_{\varepsilon, E}\right\|_{E}<\varepsilon$. Moreover, if we put $A=\{(\varepsilon, E) \mid$ $\varepsilon>0$, and $E$ is a compact set with $E \cap G p(K)=\varnothing\}$, and we define $\left(\varepsilon_{1}, E_{1}\right) \prec\left(\varepsilon_{2}, E_{2}\right)$ for $\left(\varepsilon_{i}, E_{i}\right) \in A(i=1,2)$, if $\varepsilon_{2}<\varepsilon_{1}$ and $E_{2} \supset E_{1}$, we obtain that $\lim \mu_{\varepsilon, E}=m_{\Lambda}$ in the $w^{*}$-topology of $M\left(\Gamma_{b}\right)$, where $\Gamma_{b}$ is the Bohr compactification of $\Gamma, \Lambda$ the annihilator of $G p(K)$ in $\Gamma_{b}$, and $m_{\Lambda}$ the normalized Haar measure of $\Lambda$.

Proof. The first half is obtained by modifying [9, Theorem 5] (cf. [15]). We remark that $\left\{\mu_{\varepsilon, E}\right\}_{A}$ is a net in $M(G)$. Then we have the last half by the first half. Q.E.D.

LEMMA 2. Let $K$ be an $H_{1}$-set containing 0 in $G, \varepsilon$ a positive number, and $F_{0}$ a finite subset of $G$ which is $G p(K)$-independent. Also let $E_{0}$ be a compact set in $G$ with $E_{0} \cap G p\left(F_{0} \cup K\right)=\varnothing$. Then there exists $\nu$ a probability measure in $M(\Gamma)$ such that $|\hat{\nu}-1|<\varepsilon$ on $F_{0} \cup K$ and $|\hat{\nu}|<\varepsilon$ on $E_{0}$.

Proof. Let $m_{\Lambda}$ be as in Lemma 1. By [8], for $\eta>0$, there exists $P_{0}$ a trigonometric polynomial on $\Lambda$ such that

(1) $P_{0} \geqslant 0$ on $\Lambda$ and $\left\|P_{0} m_{\Lambda}\right\|=1$,

(2) supp $\hat{P}_{0} \subset G p\left[F_{0} \cup G p(K)\right]$,

(3) $\left|\left(P_{0} m_{\Lambda}\right)-1\right|<\eta$ on $F_{0}+G p(K)$, and

(4) $\mid\left(P_{0} m_{\Lambda}\right) \hat{\mid}<$ on $E_{0}+G p(K)$. 
Choose a sufficiently large finite set $F$ with $\{0\} \cup F_{0} \subset F \subset G p\left(F_{0}\right)$ and $(F-F) \cap$ $G p(K)=\{0\}$, and set $P(\gamma)=\Sigma_{x \in F} C_{x} x(\gamma)$ on $\Gamma$, where $C_{x}=\hat{P}_{0}(x+G p(K))(x \in$ $F)$. For $\mu_{\varepsilon, E}$ as in Lemma 1, we define $\nu_{\varepsilon, E}=|P| \mu_{\varepsilon, E}$. Then by $\Gamma \subset \Gamma_{b}$ and Lemma 1 , we get that

$(5)^{\prime}\left\|\nu_{\varepsilon, E}\right\|=\int_{\Gamma_{b}}|P| d \mu_{\varepsilon, E}$,

$(6)^{\prime}\left|\int_{\Gamma_{b}}\right| P\left|d \mu_{\varepsilon, E}-\int_{\Gamma_{b}}\right| P\left|d m_{\Lambda}\right| \mapsto 0$ as $(\varepsilon, E) \mapsto \infty$, and

(7) $\left\|\nu_{\varepsilon, E}-P \mu_{\varepsilon, E}\right\|=\int_{\Gamma_{b}}|| P|-P| d \mu_{\varepsilon, E} \mapsto \int_{\Gamma_{b}} \| P|-P| d m_{\Lambda}$ as $(\varepsilon, E) \mapsto \infty$.

On the other hand, by the definition of $P$, we have $\int_{\Gamma_{b}}|P| d m_{\Lambda}=1$ and $P=|P|$ on $\Lambda$. So by $(5)^{\prime},(6)^{\prime}$ and $(7)^{\prime}$, we get that

(5) $\left\|\nu_{\varepsilon, E}\right\| \mapsto 1$ as $(\varepsilon, E) \mapsto \infty$, and

(6) $\left\|\nu_{\varepsilon, E}-P \mu_{\varepsilon, E}\right\| \mapsto 0$ as $(\varepsilon, E) \mapsto \infty$.

For the above trigonometric polynomial $P$ and for $\eta>0$, by (5), (6), the assumption of Lemmas 2 and 1 , there exists $(\varepsilon, E) \in A$ such that

(7) $E \supset\left(\left\{x_{1}-x_{2} \mid x_{1}, x_{2} \in F, x_{1} \neq x_{2}\right\}+K\right) \cup\left(E_{0}-F\right)$,

(8) $E \subset G p(K)=\varnothing$,

(9) $\left\|\hat{\mu}_{\varepsilon, E}\right\|_{E}<\eta /\left(\sum_{x \in F}\left|C_{x}\right|+1\right)$,

(10) $\mid\left\|\nu_{\varepsilon, E}\right\|-1<\eta$, and

(11) $\left\|\nu_{\varepsilon, E}-P \mu_{\varepsilon, E}\right\|<\eta$.

If we define $\nu=\nu_{\varepsilon, E} /\left\|\nu_{\varepsilon, E}\right\|$ in $M(\Gamma), \nu$ will satisfy the properties of Lemma 2. In fact, for $y \in F_{0}$ and $k \in K \cup\{0\}$, by the definition of $\nu$, we have that $\mid \hat{\nu}(y+k)-$ $1 \mid \leqslant\left(\left|\hat{\nu}_{\varepsilon, E}(y+k)-1\right|+\left|1-\left\|\nu_{\varepsilon, E}\right\|\right|\right) /\left\|\nu_{\varepsilon, E}\right\|$. By (7), (8), (9), (10), and (11), we : obtain

$$
\begin{aligned}
\left|\hat{\nu}_{\varepsilon, E}(y+k)-1\right| & \leqslant \eta+\left|\left(P \mu_{\varepsilon, E}\right)^{\wedge}(y+k)-1\right| \\
& \leqslant \eta+\left|C_{y} \hat{\mu}_{\varepsilon, E}(k)-1\right|+\sum_{\substack{x \in F \\
x \neq y}}\left|C_{x}\right|\left|\hat{\mu}_{\varepsilon, E}(y+k-x)\right| \\
& \leqslant \eta+2 \eta+\eta,
\end{aligned}
$$

and $|\hat{\nu}(y+k)-1| \leqslant(5 \eta) /(1-\eta)$. Also for $e \in E_{0}$, we have that

$$
|\hat{\nu}(e)| \leqslant\left(\eta+\left|\left(P \mu_{\varepsilon, E}\right)^{\wedge}(e)\right|\right) /\left\|\nu_{\varepsilon, E}\right\|
$$

and $|\hat{\nu}(e)| \leqslant(2 \eta) /(1-\eta)$ by (7) and (8). Thus $\nu$ satisfies what we want for or sufficiently small $\eta$. Q.E.D.

Proof of Theorem 1. Let $\mu$ be any measure in $M(G)$. By $\left|\hat{\mu}\left(h_{K}\right)\right|=\left|\left(P_{K} \mu \hat{)(1)} \mid\right)\right|$ and the Proposition, it will suffice to show $\left\|\left(P_{K} \mu\right)^{\hat{\imath}}\right\|_{\infty} \leqslant\|\hat{\mu}\|_{\infty}$ for all $\mu \in M(G)$. i $)$. For $\varepsilon>0$, there exists $F$ a finite set such that

(1) $\left\|\nu_{0}-P_{K} \mu\right\|<\varepsilon$, where $\nu_{0}=\left.\mu\right|_{G p(K)+F}$.

Let $k \in K$ be fixed. Since $K$ is an $H_{1}$-set, $K-\{k\}$ is an $H_{1}$-set with $K-\{k\} \ni 0$ in in $G$. Then there exists $F_{0}$ a finite set in $G$ such that $F_{0}$ is $G p(K-\{k\})$-independent int and $G p(F \cup K)=G P\left(F_{0} \cup(K-\{k\})\right)$. So there exists $N$ a natural number such ich that

(2) $\left\|\tau-\nu_{0}\right\|<\varepsilon$, where $\tau=\left.\mu\right|_{K_{N}}$, and

$$
K_{N}=N\left(F_{0} \cup(K-\{k\}) \cup\left(-F_{0}\right) \cup(-K+\{k\})\right) .
$$


Now let $E_{0}$ be a compact subset of $G$ such that

(3) $\left\|\left.(\mu-\tau)\right|_{G \backslash E_{0}}\right\|<\varepsilon$ and $E_{0} \cap G p\left(F_{0} \cup(K-\{k\})\right)=\varnothing$.

By Lemma 2, there exists $\nu$ a probability measure in $M(\Gamma)$ such that

(4) $\|\hat{\nu}-1\|_{F_{0} \cup(K-\{k\})}<\varepsilon / N$, and

(5) $\|\hat{\nu}\|_{E_{0}}<\varepsilon$.

By (1), (2), and (3), we get that

$$
\left|\int_{G} \hat{\nu}(x) \gamma(x) d \mu(x)\right| \geqslant\left|\int_{K_{N}} \hat{\nu}(x) \gamma(x) d \mu(x)\right|-\left|\int_{E_{0}} \hat{\nu}(x) \gamma(x) d \mu(x)\right|-3 \varepsilon .
$$

Also by (4) and (5), we have that $|\hat{\nu}(x)-1|<\varepsilon$ for $x \in K_{N}$, and

$$
\left|\int_{G} \hat{\nu}(x) \gamma(x) d \mu(x)\right|<|\hat{\tau}(\gamma)|-\varepsilon-\varepsilon-3 \varepsilon .
$$

On the other hand, we have that $\left|\int_{G} \hat{\nu}(x) \gamma(x) d \mu(x)\right| \leqslant\|\hat{\mu}\|_{\infty}\|\nu\|=\|\hat{\mu}\|_{\infty}$. Then we get that $|\hat{\tau}(\gamma)| \leqslant\|\hat{\mu}\|_{\infty}+5 \varepsilon$. Thus by (1) and (2), we have that $\left|\left(P_{K} \mu\right)(\gamma)\right| \leqslant$ $\|\hat{\mu}\|_{\infty}+7 \varepsilon$ and $\left\|\left(P_{K} \mu\right)\right\|_{\infty} \leqslant\|\hat{\mu}\|_{\infty}$.

The last half is as follows. By Körner [6], there exist $K$ a perfect independent $H_{\alpha}$-set $(0<\alpha<1)$ and a probability measure $\sigma \in M(K)$ such that $\varlimsup_{\gamma \rightarrow \infty}|\hat{\sigma}(\gamma)|<$ 1. If we assume $h_{K} \in \bar{\Gamma}$, there exist $\left\{\gamma_{n}\right\}$ such that $\lim _{n \rightarrow \infty} \int \gamma_{n} d \sigma=\int h_{K} d \sigma$. Since we have that

$$
\lim _{n \rightarrow \infty}\left|\int \gamma_{n} d \sigma\right|=\varlimsup_{\gamma \rightarrow \infty}|\sigma(\gamma)|<1
$$

and $\int h_{K} d \sigma=1$ by the property of $\sigma$, we get the contradiction. Q.E.D.

LEMMA 3 [7, p. 114]. Let $G$ be anondiscrete LCA group, and L a totally disconnected perfect independent set and non-Helson set in $G$. Then there exists $K \subset L$ a clopen non-Helson set in the relative topology of $L$ such that $\left\|\hat{\mu}_{\mid G p(K)}\right\|_{\infty} \geqslant 3\|\hat{\mu}\|_{\infty}$ for some nonzero measure $\mu$ in $M(L)$.

LEMma 4 [9]. Let $G$ be a nondiscrete LCA group, $F \subset G$ a finite set, and $E \subset G$ a closed subset with $E \cap G p(F)=\varnothing$. Then to each $\varepsilon>0$, there exists $f \in A(G)=$ $L^{\prime}(\Gamma)$ such that $0 \leqslant f \leqslant 1$ on $G, f=1$ on $F, f=0$ on some neighborhood of $E$, and $\|f\|_{A(G)}<1+\varepsilon$.

Proof of Theorem 2. Since $G$ is a nondiscrete LCA group, there exists $L$ a totally disconnected perfect independent set and non-Helson set such that

$$
\underset{\substack{\gamma \rightarrow \infty \\ \gamma \in \Gamma}}{\lim }\|\gamma-1\|_{L}=0
$$

(cf. $[5,8])$. Then by Lemma 3 , there exists $K \subset L$ a perfect subset with the property of Lemma 3. Now we shall show $h_{K} \notin \bar{\Gamma}$. By the Proposition, it is sufficient to show that we get a contradiction if we assume $\left\|\left(P_{K} \nu\right)\right\|_{\infty} \infty\|\hat{\nu}\|_{\infty}$ for all $\nu \in M(G)$. We define $K^{\prime}=L \backslash K$, and for $\mu$ as in Lemma $3, P_{K} \mu=\mu_{1}+\mu_{2}$ where $\mu_{1}=\mu_{\mid K}$ and $\mu_{2}=\mu_{1 K^{\prime}}$. Since $L$ is an independent set, we have that (a) $\mu_{1}=\mu_{\mid G p(K)}$, and (b) $\mu_{2} \in M_{d}\left(K^{\prime}\right)$. By (a) and the choice of $\mu$, we get $\left\|\hat{\mu}_{1}\right\|_{\infty}=\left\|\hat{\mu}_{\mid G p(K)}\right\|_{\infty} \geqslant 3\|\hat{\mu}\|_{\infty}$. 
Also by (b), we assume that the support of $\mu_{2}$ is a finite set. By Lemma 4, to each $\varepsilon>0$, there exists $f \in A(G)(0 \leqslant f \leqslant 1)$ such that $f=1$ on supp $\mu_{2}, f=0$ on $K$, and $\|f\|_{\left.A()_{i}\right)}<1+\varepsilon$. Then for $\gamma \in \Gamma$, we have that

$$
\int_{G} \gamma f d\left(P_{K^{\prime}} \mu\right)=\int_{K^{\prime}} \gamma f d\left(P_{K^{\prime}} \mu\right)+\int_{K^{\prime}} \gamma f d\left(P_{K} \mu\right)=\int_{K^{\prime}} \gamma f d\left(P_{k} \mu\right)
$$

Thus we get that

$$
\begin{aligned}
& \left|\int_{G^{\prime}} \gamma d \mu_{2}\right|=\left|\int_{K^{\prime}} \gamma f d\left(P_{K^{\prime}} \mu\right)\right|=\left|\int_{G^{\prime}} \gamma f d\left(P_{K^{\prime}} \mu\right)\right| \\
& \leqslant\|f\|_{A\left(C_{i}\right)}\left\|\left(P_{K} \mu\right)^{\hat{n}}\right\|_{\infty}<(1+\varepsilon)\left\|\left(P_{K} \mu\right)^{\hat{n}}\right\|_{\infty} .
\end{aligned}
$$

and $\left\|\hat{\mu}_{2}\right\|_{\infty} \leqslant\left\|\left(P_{K} \mu\right)\right\|_{\infty}\left(\leqslant\|\hat{\mu}\|_{\infty}\right)$. On the other hand, by the assumption of the contradiction and the property of Lemma 3, we have that

$$
\begin{aligned}
\|\hat{\mu}\|_{\infty} & \geqslant\left\|\hat{\mu}_{1}\right\|_{\infty}-\left\|\hat{\mu}_{2}\right\|_{\infty}=\left\|\hat{\mu}_{\mid(i p, K)}\right\|_{\infty}-\left\|\hat{\mu}_{2}\right\|_{\infty} \\
& \geqslant 3\|\hat{\mu}\|_{\infty}-\|\hat{\mu}\|_{\infty}=2\|\hat{\mu}\|_{\infty} .
\end{aligned}
$$

Therefore we get a required contradiction. Q.E.D.

Proof of Corollary 1. By the well-known fact [7]. there exists $K$ a totally disconnected perfect independent set with $\sigma(\neq 0) \in M(K)$ and $\hat{\sigma}(\gamma) \rightarrow 0$ as $\gamma \rightarrow \infty$. Then $K$ is divided with $K=K_{1} \cup K_{2}$ such that $K_{1} \cap K_{2} \neq \varnothing$ and $K_{1}, K_{2}$ are nonempty perfect compact subsets with $\tau(\neq 0) \in M\left(K_{1}\right)$ and $\hat{\tau}(\gamma) \mapsto 0$ as $\gamma \mapsto \propto$. Since $K$ is an independent set, $\left\{K_{1}+\{x\}\right\}_{x \in K_{2}}$ are pairwise disjoint sets. By [11], there exists $K(x)$ a totally disconnected perfect independent and non-Helson set with $K(x) \subset K_{1}+\{x\}$. Then $\{K(x)\}_{x \in K_{2}}$ are an uncountable set, and $\left\{h_{K(x)}\right\}_{x \in K_{2}}$ are what we require. In fact, we assume $x \neq y$ and $K(x) \cap(G p(K(y))+z) \neq \varnothing$. Hence, if there exist $w, w^{\prime} \in K(x)$ such that $w=z+\sum_{i} n_{i} k(y, i)$ and $w^{\prime}=z+$ $\sum_{1} n_{i}^{\prime} k^{\prime}(y, i)$ where $n_{i}, n_{n}^{\prime}$ integers and $k(y, i), k^{\prime}(y, i) \in K(y)$, we have $w-w^{\prime}=$ $-\sum_{i} n_{i}^{\prime} k_{i}(y, i)+\sum_{i} n_{i} k(y, i)$. Since we have $G p(K(x)) \cap G p(K(y))=\{0\}$ by $x \neq y$, we get $w=w^{\prime}$.

Thus there exists $\mu$ a continuous probability measure on $K(x)$ such that $\hat{\mu}\left(h_{K(y)}\right)$ $=0$. Therefore we proved the first half. Also it is well known that there exists $K$ a totally disconnected perfect independent $H_{1}$-set in $G$. So the last half can be proved in the same way as the above method by Theorem 2. Q.E.D.

The proof of Corollary 2 is clear by Theorem 2 and [13]."

3. $H$-Kronecker set. In this section, we show a generalization of [12] in some sense. First we define $H$-Kronecker set.

Definition. Let $G$ be a nondiscrete LCA group, $K$ a compact set, and $H$ a closed subgroup of $G$. $K$ is called an $H$-Kronecker set if, to each $\varepsilon>0$ and $f \in C(K)$ with $|f|=1$, there exists $\gamma \in H^{\perp}$ such that $\|f-\gamma\|_{K}<\varepsilon$. We simply call $K$ a Kronecker set. if $H=\{0\}$.

Next we shall show the existence of a nontrivial $H$-Kronecker set. 
ThEOREM 3. Let $G$ be a metric I-group, and $H$ a o-compact closed subgroup with Haar measure zero such that $\{p x: x \in U\} \not \subset H$ for any natural number $p$ and for any neighborhood $U$ of $0 \in G$. Then there exists $K$ an $H$-Kronecker set which is a totally disconnected perfect set.

The proof is obtained by modifying $[8,5.2]$.

THEOREM 4. Under the above assumption, let $\rho$ be a nonzero continuous measure on $K$. Then we have that for any $\gamma \in \Gamma, \mu_{i} \in M(G)(1 \leqslant i \leqslant n)$, and $\varepsilon>0$, there exists $\gamma_{0} \in \Gamma$ such that $\left.\mid\left(h_{H} \mu_{i}\right) \hat{(} \gamma\right)-\left(h_{H} \mu_{i}\right)\left(\gamma_{0}\right) \mid<\varepsilon(1 \leqslant i \leqslant n)$ and $\left|\hat{\rho}\left(\gamma_{0}\right)\right|>\|\rho\| / 2$.

Proof. Let $\pi$ be the natural mapping from $G$ to $G / H$, and $\check{\pi}: M(G) \mapsto M(G / H)$ the mapping induced by $\pi$. Then we remark $\left.\check{\pi}(\mu) \hat{\mu} \hat{\mu}\right|_{H^{\perp}}$ for all $\mu \in M(G)$. Now by the definition of $K, \pi(K)$ is a Kronecker set on $G / H$. Hence, by an application of [12] in $G / H$, there exists $\gamma_{0} \in H^{\perp}$ such that

$$
\left|\check{\pi}\left(\bar{\gamma} h_{H} \mu_{i}\right) \hat{(1)}-\check{\pi}\left(\bar{\gamma} h_{H} \mu_{i}\right) \hat{(}\left(\gamma_{0}\right)\right|<\varepsilon \quad(1 \leqslant i \leqslant n),
$$

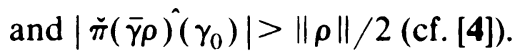

That is, we have

$$
\left.\mid\left(h_{H} \mu_{i}\right) \hat{(\gamma}\right)-\left(h_{H} \mu_{i}\right) \hat{(}\left(\gamma_{0}+\gamma\right) \mid<\varepsilon \quad(1 \leqslant i \leqslant n) .
$$

and $\left|\hat{\rho}\left(\gamma_{0}+\gamma\right)\right|>\|\rho\| / 2$. This establishes the result. Q.E.D.

\section{REFERENCES}

1. (j. Brown, Idempotents in the closure of the characters, Bull. London Math. Soc. 4 (1972), 43-46.

2. C. F. Dunkl and D. E. Ramirez, Locally compact subgroup of the spectrum of the measure algebra. Semigroup Forum 3 (1971), 95-107.

3. $122-126$ . Bounded projections on Fourier-Stieltjes transform. Proc. Amer. Math. Soc. 31 (1972),

4. I. Glicksberg and I. Wik. The range of Fourier-Stieltjes transforms of parts of measures. Conference on Harmonic Analysis. Maryland (1971), Lecture Notes in Math., vol. 266, Springer-Verlag, Berlin and New York, 1972.

5. C. C. Graham and O. C. McGehee, Essal's in commutative harmonic analysis, Springer-Verlag. Berlin and New York, 1979.

6. T. W. Körner, Some results on Kronecker, Dirichlet and Helson sets. II, J. Analyse Math. 27 (1974). $260-388$.

7. L. A. Lindahl and F. Poulsen (editors), Thin sets in harmonic analysis, Marcel Dekker. New York, 1972.

8. W. Rudin, Fourier analysis on groups, Interscience Tracts No. 12, Interscience, New York, 1962.

9. S. Saeki, On the union of tw'o Helson sets, J. Math. Soc. Japan 23 (1971), 636-648.

10. On strong Ditkin sets, Ark. Mat. 10 (1972), 1-7.

11. Symmetric maximal ideals in $M(G)$, Pacific J. Math. 54 (1974), 229-243.

12. Bohr compactification and continuous measure. Proc. Amer. Math. Soc. 80 (1980), 244-246

13. S. Saeki and E. Sato, Critical points and point derivations on $M(G)$. Michigan Math. J. 25 (1978), $147-161$.

14. J. L. Taylor, Measure algebras, CBMS Regional Conf. Ser. in Math., no. 16. Amer. Math. Soc.. Providence, R.I., 1973.

15. N. Th. Varopoulos, Groups of continuous functions in harmonic analysis, Acta Math. 125 (1970), $109-152$. 